\title{
Thrombotic Risk of Rotor Speed Modulation Regimes of Contemporary Centrifugal Continuous-flow Left Ventricular Assist Devices
}

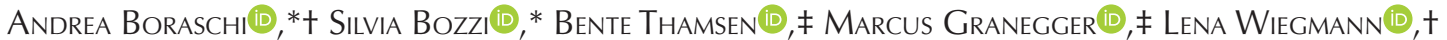

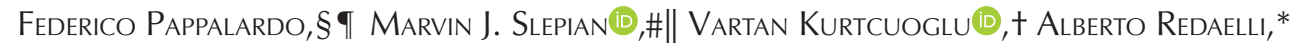 \\ Diane De Zélicourtid, † and Filippo Consolo@
}

\begin{abstract}
Contemporary centrifugal continuous-flow left ventricular assist devices (LVADs) incorporate dynamic speed modulation algorithms. Hemocompatibility of these periodic unsteady pump operating conditions has been only partially explored. We evaluated whether speed modulation induces flow alterations associated with detrimental prothrombotic effects. For this aim, we evaluated the thrombogenic profile of the HeartWare ventricular assist device (HVAD) Lavare Cycle (LC) and HeartMate3 (HM3) artificial pulse (AP) via comprehensive numerical evaluation of (i) pump washout, (ii) stagnation zones, (iii) shear stress regimens, and (iv) modeling of platelet activation status via the platelet activity state (PAS) model. Data were compared between different simulated operating scenarios, including: (i) constant rotational speed and pump pressure head, used as reference; (ii) unsteady pump pressure head as induced by cardiac pulsatility; and (iii) unsteady rotor speed modulation of the LC (HVAD) and AP (HM3). Our results show that pump washout did not improve across the different simulated scenarios in neither the HVAD nor the HM3. The LC reduced but did not eliminate flow stagnation
\end{abstract}

From the *Department of Electronics, Information and Bioengineering, Politecnico di Milano, Milano, Italy; +The Interface Group, Institute of Physiology, University of Zurich, Zurich, Switzerland; ‡Biofluid Mechanics Laboratory, Institute for Imaging Science and Computational Modelling in Cardiovascular Medicine, Charité-Universitätsmedizin Berlin, Berlin, Germany; §Univeristà Vita Salute San Raffaele, Milano, Italy; Department of Anesthesia and Intensive Care, IRCCS ISMETT, UPMC Italy, Palermo, Italy; \|Sarver Heart Center, University of Arizona, Tucson, Arizona; and \#Department of Biomedical Engineering, University of Arizona, Tucson, Arizona.

Submitted for consideration May 2020; accepted for publication in revised form August 2020.

Disclosure: The authors have no conflicts of interest to report.

This work was supported by Fondazione Cariplo (Milano, Italy; grant 2015-1044 awarded to FC and MJS), is part of the Zurich Heart project under the umbrella of "Hochschulmedizin Zürich" and is supported by the Stavros Niarchos Foundation.

HM3, HeartMate3 ventricular assist device; HVAD, HeartWare ventricular assist device; IQR, interquartile range; PAS, platelet activation state.Supplemental digital content is available for this article. Direct URL citations appear in the printed text, and links to the digital files are provided in the HTML and PDF versions of this article on the journal's Web site (www.asaiojournal.com).

DDZ and FC have contributed equally to this work.

Correspondence: Filippo Consolo, PhD, Università Vita Salute San Raffaele, Via Olgettina 58, 20132 Milano, Italy. Email: consolo. filippo@unisr.it; Diane de Zélicourt, The Interface Group, Institute of Physiology, University of Zurich, Winterthurerstrasse 190, $\mathrm{CH}-8057$ Zürich, Switzerland. Email: diane.dezelicourt@physiol.uzh.ch.

Copyright (C) ASAIO 2020

DOI: 10.1097/MAT.0000000000001297
$(-57 \%)$ and did not impact metrics of HVAD platelet activation (median PAS: $+\mathbf{+ 0 . 4 \%} \%$ ). The AP reduced HM3 flow stagnation by up to $91 \%$ but increased prothrombotic shear stress and simulated platelet activation (median PAS: $+124 \%$ ). Our study advances understanding of the pathogenesis of LVAD thrombosis, suggesting mechanistic implications of rotor speed modulation. Our data provide rationale criteria for the future design optimization of next generation LVADs to further reduce hemocompatibility-related adverse events. ASAIO Journal 2020; XX:00-00.

Key Words: left ventricular assist device, HVAD Lavare Cycle, HeartMate 3 artificial pulse, thrombosis

Third generation centrifugal continuous-flow left ventricular assist devices (LVADs) have achieved significant improvement in long-term clinical efficacy compared with prior designs, nearing heart transplantation in many respects. ${ }^{1}$ However, these devices remain limited by hemocompatibility-related adverse events (HRAEs), including device thrombosis, thromboembolic complications, and bleeding events. ${ }^{2,3}$ Previous studies have linked HRAEs to the abnormal hemodynamic profiles and shear stresses generated within the LVAD. Shearmediated platelet activation and degradation of high molecular weight von Willebrand factor multimers have been identified as drivers mediating the development of thrombotic and bleeding events. ${ }^{4-9}$ Concomitantly, antithrombotic agents routinely administered to LVAD patients have been demonstrated to be largely ineffective, mechanistically, in limiting shear-mediated platelet activation. ${ }^{10,11}$ This paradigm has been further supported by clinical data revealing that the thrombotic risk is not completely suppressed by current therapeutic pharmacological strategies. ${ }^{12}$ On the other hand, intense anticoagulation has been suggested to possibly exacerbate bleeding risk. ${ }^{13}$

In this scenario, accurate mechanistic characterization of how and to what extent an LVAD interacts with and alters physiologic hemostasis is crucial for rationale design of next generation devices to further improve hemocompatibility and reduce adverse events

Computational fluid dynamics (CFD) simulations provide a unique means to characterize hemodynamic patterns and shear stresses in cardiovascular devices and have been extensively applied to evaluate the thrombogenic potential of different elements of the LVAD system, including (i) the pump, ${ }^{14-16}$ (ii) the inflow cannula, ${ }^{17}$ (iii) the LVAD-implanted ventricle, $^{18}$ (iv) the outflow graft, ${ }^{19,20}$ and (v) the anastomosed aorta. ${ }^{21}$ However, the oversimplified LVAD operating 
conditions simulated in previous studies have limited their translational impact. Influence of the dynamic pump pressure head, as provided by clinically observed residual ventricular contractility, has been accounted for in only a few studies. ${ }^{16,18}$ Furthermore, evaluation of the effect of unsteady rotor speed modulation regimes on hemocompatibility has been only partially explored, ${ }^{16}$ and no data are available related to prothrombotic platelet activation induced by speed modulation.

Rotor speed modulation consists of cyclic acceleration and deceleration of the rotating LVAD impeller with respect to a set baseline rotational speed. ${ }^{15,22}$ These dynamic rotation sequences have been introduced to improve pump and ventricular washout by reducing blood stasis, as well as to restore some pulsatility in the aorta. ${ }^{23,24}$

We hypothesized that, despite providing a modicum of washout and pulsatility, cyclic acceleration and deceleration of the rotating impeller of the pump may be detrimental, contributing to the generation of unfavorable, prothrombotic hemodynamic conditions associated with sudden, high magnitude, and highly oscillating shear stress within the pump. Our hypothesis stems from and is consistent with earlier work, wherein we exposed platelets to shear stress fluctuations representative of those experienced in cardiovascular devices and demonstrated that shear stress dynamicity, that is, oscillations of shear stress over time, was a major determinant of the measured prothrombotic platelet activation, with important implications for the development of device-related thrombotic events. ${ }^{25}$

To test our hypothesis, we coupled, for the first time, mechanistic analysis of prothrombotic features of the LVAD hemodynamics with metrics of shear-mediated platelet activation under unsteady_clinically relevant_LVAD operating scenarios.

\section{MATERIALS AND METHODS}

We conducted a comprehensive analysis of the hemodynamic and thrombogenic profile of currently most used commercial LVADs that both feature a rotor speed modulation sequence, namely: (i) the HeartWare HVAD (Medtronic, Minneapolis, MN) with the Lavare Cycle (LC), and (ii) the HeartMate3 (HM3, Abbott, Chicago, IL) with the artificial pulse (AP). Using CFD simulations, we simulated blood flow within the pumps associated with different LVAD operating scenarios, including:

(i) constant operating conditions, namely constant rotational speed and pump pressure head;

(ii) the unsteady hemodynamic environment (i.e., unsteady pressure head) induced by isolated effect of cardiac pulsatility imposing the pressure pulsations typical of heart failure patients $(10 \%$ residual left ventricular ejection fraction) with speed modulations still turned off;

(iii) the unsteady hemodynamic environment (i.e., unsteady rotational speed and resulting unsteady pressure head) induced by isolated effect of rotor speed modulation sequences, namely the LC of the HVAD and the AP of the HM3.

HVAD and HM3 geometries (Figure 1) were obtained from Thamsen et al. ${ }^{26}$ and Wiegmann et al., ${ }^{16}$ respectively. Evaluation of pump thrombogenicity was based on postprocessing analysis of CFD data, using combined information gained from the internal pump hemodynamic profile, shear stress regimes, and metrics of platelet activation. In detail, we characterized

(i) pump washout, that is, the time to remove a passive scalar from the device by simple advection; the passive scalar represents the relative concentration of a dye crossing the pump;
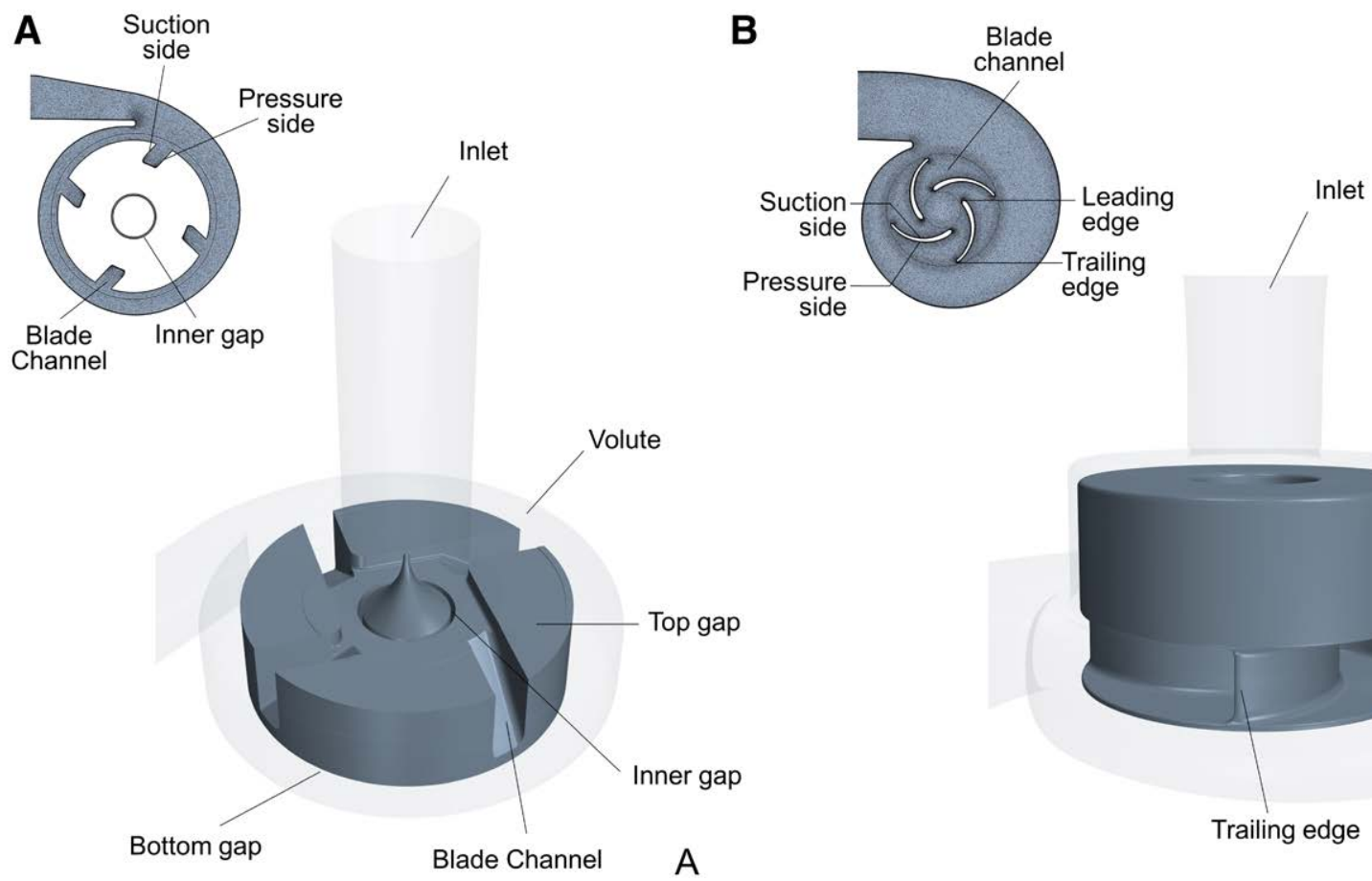

Figure 1. Geometries of the HVAD (A) and HM3 (B). Pump housing and impeller are shown in light and dark gray, respectively. Insets on the top left of $(A)$ and $(B)$ show a cross-sectional view of the polyhedral mesh used for the simulations. HVAD, HeartWare ventricular assist device; HM3, HeartMate3. 

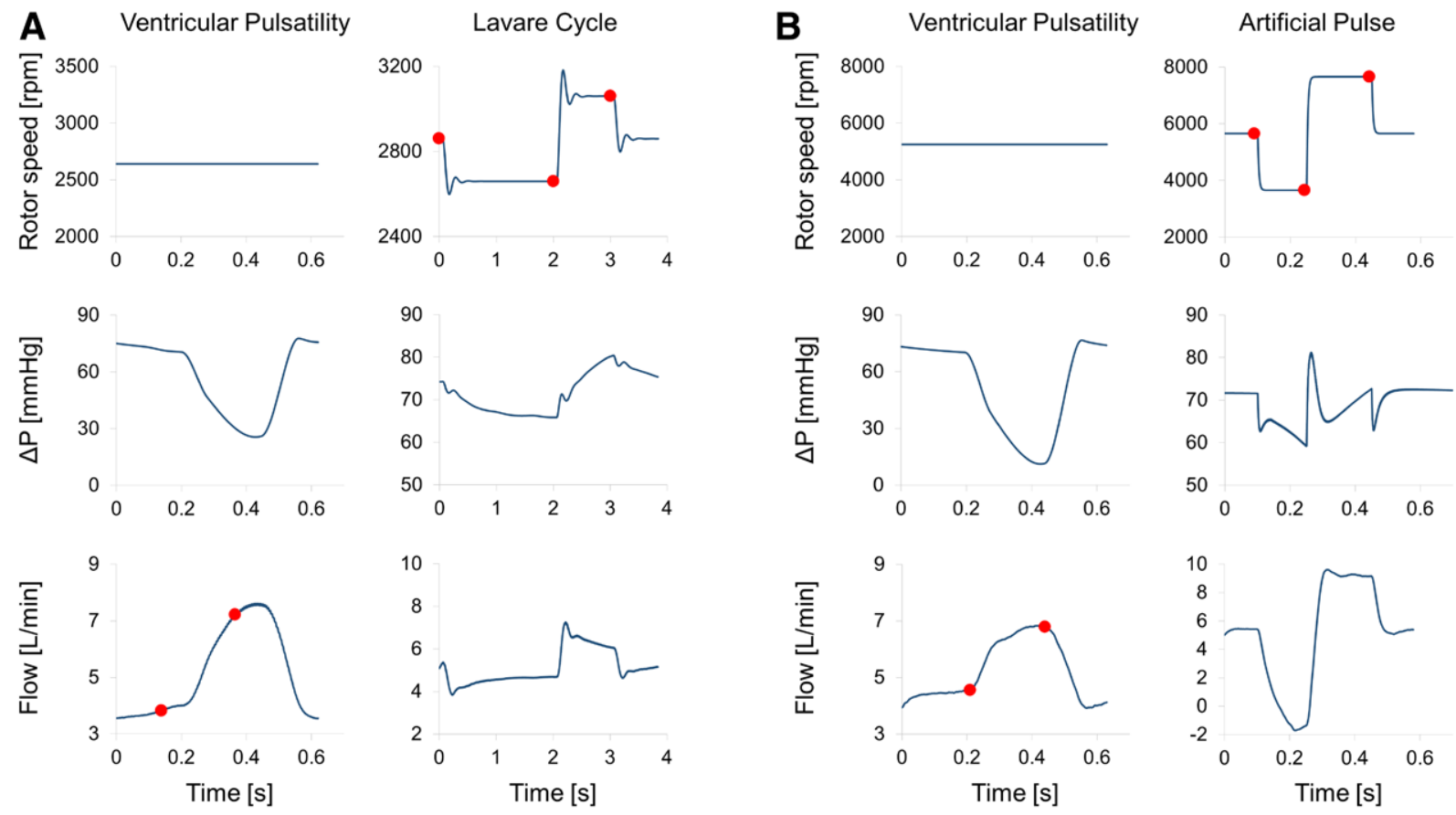

Figure 2. Profiles of pump rotational speed and pressure head imposed as boundary conditions in CFD simulations reproducing residual ventricular pulsatility (10\% ejection fraction) and speed modulation sequences in the HVAD (A) and HM3 (B). Flow rate waveforms obtained from CFD simulations are also shown. Red dots indicate time instants of injection of platelet-like particles for Lagrangian particle tracking. CFD, computational fluid dynamics; HM3, HeartMate3; HVAD, HeartWare ventricular assist device.

(ii) volumes of flow stagnation, defined as internal regions of the pump with blood velocities $<0.2 \mathrm{~m} / \mathrm{s}$ and further than $40 \mu \mathrm{m}$ away from the static housing or rotating impeller surfaces;

(iii) fluid shear stress, which includes both viscous and turbulent stress components;

(iv) shear-mediated platelet activation status, computed according to the numerical platelet activity state (PAS) model. ${ }^{25}$ PAS values were derived by tracking over time the fluid shear stress experienced by simulated plateletlike particles flowing through the pump..$^{25}$ Platelet-like particles were injected in the pump at time instants indicated in Figure 2.

Details on settings for the CFD simulations are reported in the Supplementary Materials, Supplemental Digital Content 1 (http://links.Iww.com/ASAIO/A564).

\section{RESULTS}

\section{Pump Washout}

Scalar washout and flow structures within the two pumps are shown in Figure 3, for operation under constant rotational speed and pressure head. As blood enters the HVAD, it impinges upon the blades, forming a confined stream of new blood on the pressure side (dark blue in Figure $\mathbf{3 A}, \mathbf{I}$ ), and large flow detachment and recirculation zones in the blade channels on the suction side (Figure 3A, II). In contrast, blood flow through the HM3 shows limited flow separation and rather uniform washout in the blade channels (Figure 3B, IV). These differences notwithstanding, both the HVAD and HM3 revealed efficient and comparable pump washout, primarily dictated by pump size and flow rate. Time required for $95 \%$ washout under constant operating conditions was 0.34 and $0.29 \mathrm{sec}$ in the HVAD and HM3, respectively. As the mean pump flow was matched across all tested conditions, ventricular pulsations, and speed modulation did not significantly affect the 95\% washout time: the 95\% washout time slightly decreased to $0.28 \mathrm{sec}$ during the acceleration phase of the HVAD LC; the HM3 AP led to a transient flow reversal during deceleration, with blood flowing back toward the ventricle, followed by rapid acceleration, slightly increasing the $95 \%$ washout time to $0.32 \mathrm{sec}$. Across all tested conditions, regions that took longest to washout in the HVAD were the secondary flow paths through bottom and inner gaps (Figure 3, A and C, III). Washout of the secondary flow path through the top gap was slower than the main flow pathway in the HM3 as well (Figure 3, B and D, V), but washout patterns were generally more uniform and differences less marked.

\section{Flow Stagnation}

Both pumps revealed flow stagnation zones indicative of possible prothrombotic risk. Consistent with the scalar washout fields, stagnation zones in the HVAD at constant speed and fixed pressure head were observed in the flow detachment region within the blade channels (Figure $\mathbf{4 A}, \mathrm{I}$ ), in the bottom and inner gaps (Figure 4A, II), and in the outlet duct of the volute (Figure 4A, III). Under constant operating conditions, stagnation zones in the HM3 were observed at the junction of the inlet cannula to the volute (Figure 4D, IV), within the secondary flow path in the top gap (Figure 4D, V), and centrally under the rotor (Figure 4D, VI) as well as in the outlet duct of the volute (Figure 4D, VII). Comprehensively, $\sim 1 \%$ of the HVAD fluid 


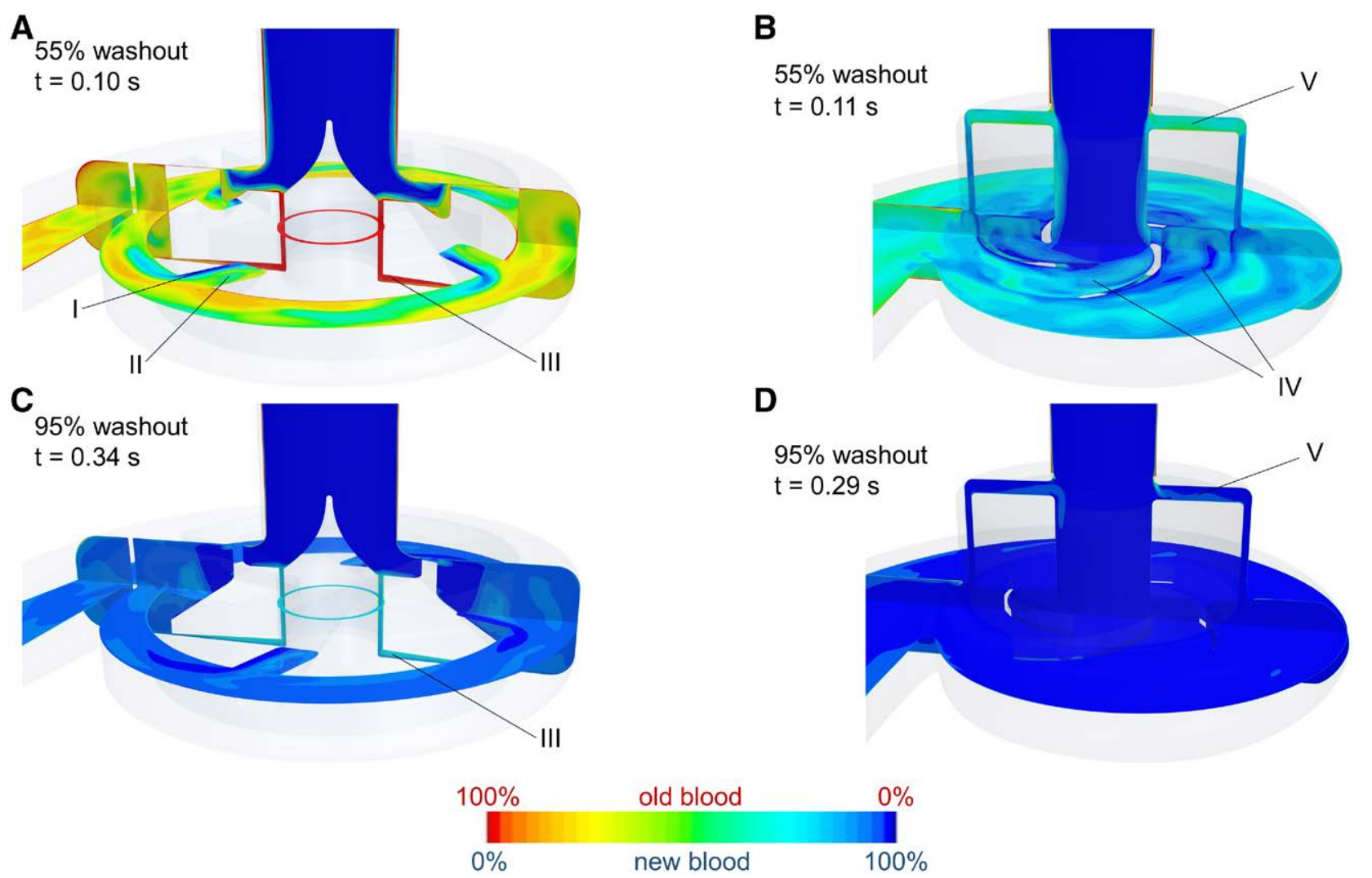

Figure 3. Comparison of flow structures and washout performance in the HVAD (A: $55 \%$ washout, C: $95 \%$ washout) and HM3 (B: $55 \%$ washout, D: $95 \%$ washout) under constant rotational speed and pressure head. HM3, HeartMate3; HVAD, HeartWare ventricular assist device.
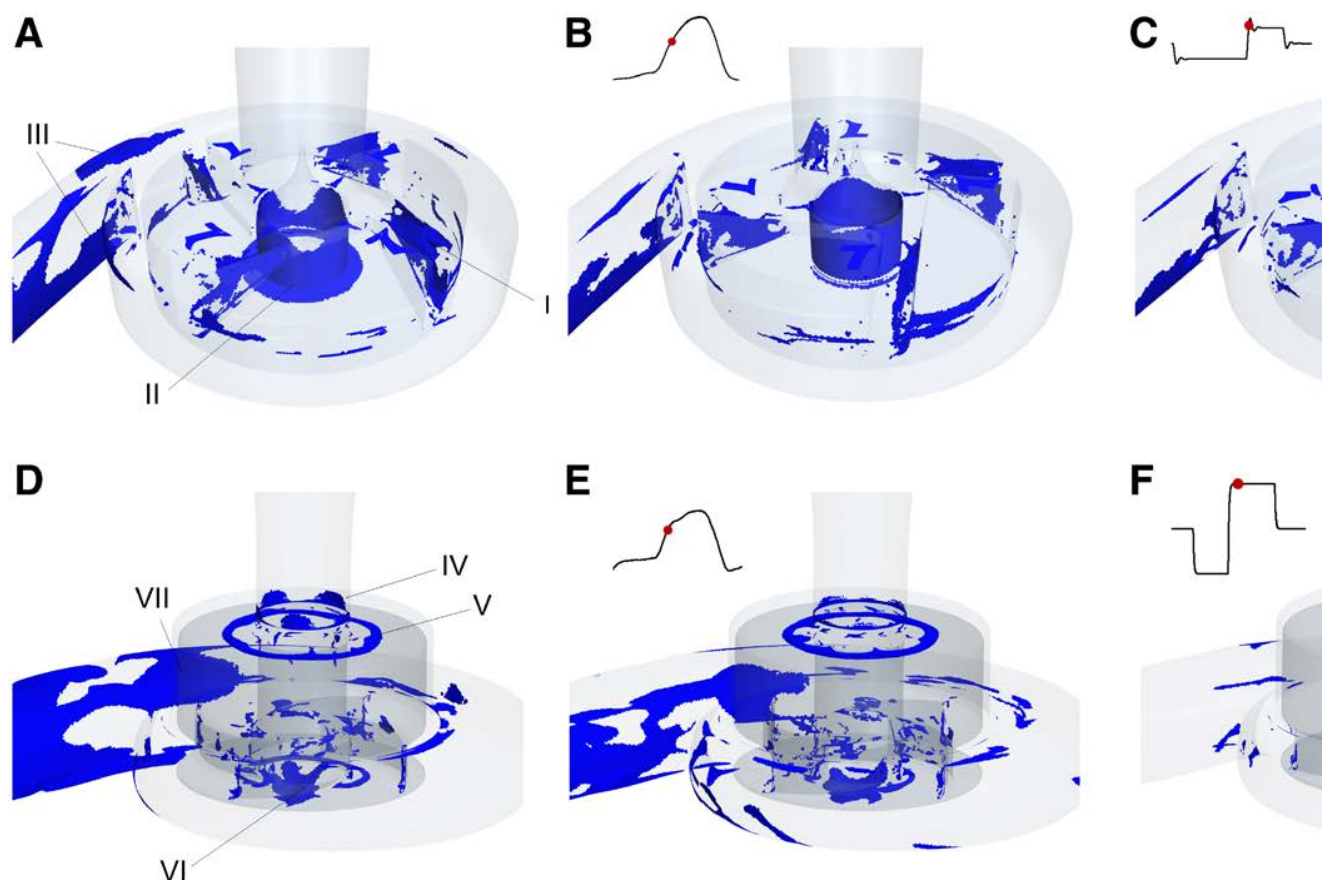

$\mathbf{E}$
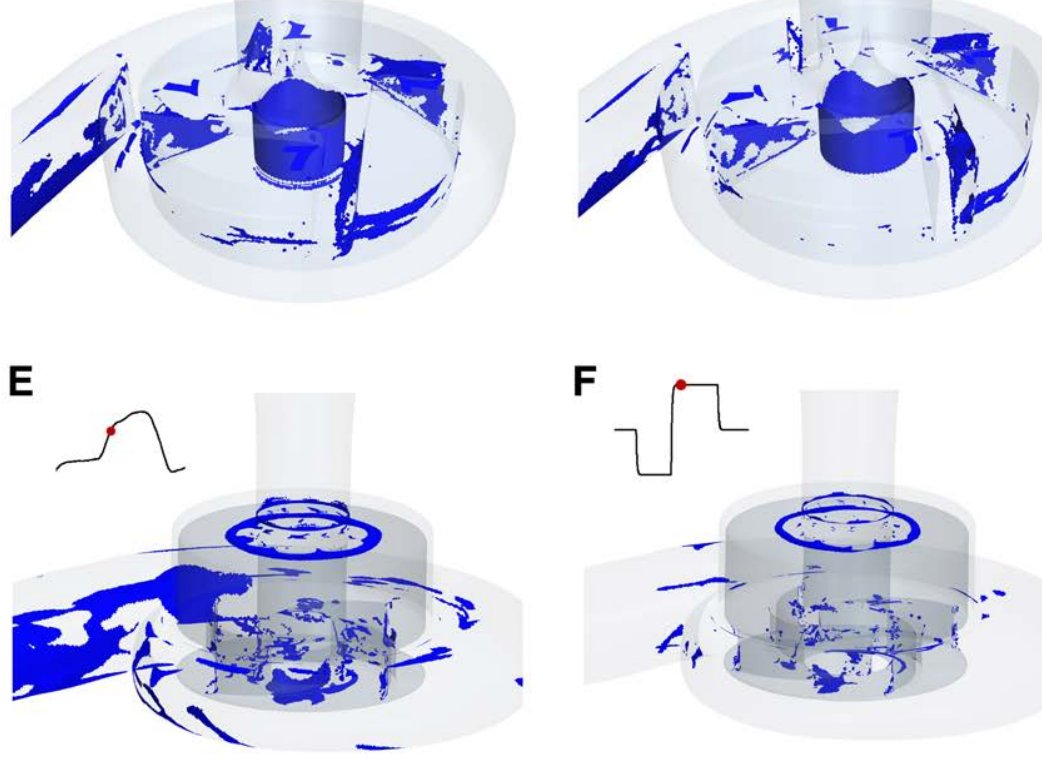

$\mathbf{F}$

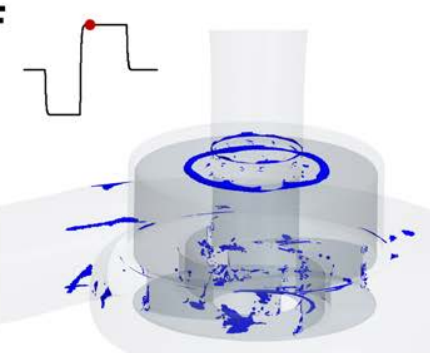

Figure 4. Flow stagnation zones within the HVAD and HM3 under static operating conditions (A, D), ventricular pulsatility (B, E) and speed modulation sequences (C, F). HM3, HeartMate3; HVAD, HeartWare ventricular assist device. 
Table 1. Computed Differences in Pump Hemodynamics and Metrics of Platelet Activation Across the Different Simulated Conditions for the Two Pumps

\begin{tabular}{|c|c|c|c|c|c|c|}
\hline & \multicolumn{3}{|c|}{ HVAD } & \multicolumn{3}{|c|}{ HM3 } \\
\hline & Baseline & $\begin{array}{l}\text { Ventricular } \\
\text { Pulsatility }\end{array}$ & Lavare Cycle & Baseline & $\begin{array}{l}\text { Ventricular } \\
\text { Pulsatility }\end{array}$ & Artificial Pulse \\
\hline $\begin{array}{l}\text { Volume of flow stagnation } \\
\text { (\% of total volume) }\end{array}$ & $0.85 \%$ & $0.35 \%$ & $0.36 \%$ & $3.60 \%$ & $1.73 \%$ & $0.30 \%$ \\
\hline $\begin{array}{l}\text { Proportion of platelets exposed } \\
\text { to shear stress }>50 \mathrm{~Pa}\end{array}$ & $78 \%$ & $82 \%$ & $75 \%$ & $52 \%$ & $59 \%$ & $92 \%$ \\
\hline PAS values (median and IQR) & $\begin{array}{c}0.077 \\
(0.047-0.146)\end{array}$ & $\begin{array}{c}0.084 \\
(0.051-0.144)\end{array}$ & $\begin{array}{c}0.077 \\
(0.045-0.149)\end{array}$ & $\begin{array}{c}0.046 \\
(0.030-0.079)\end{array}$ & $\begin{array}{c}0.049 \\
(0.030-0.082)\end{array}$ & $\begin{array}{c}0.104 \\
(0.071-0.149)\end{array}$ \\
\hline
\end{tabular}

Volumes of flow stagnation depict the average baseline stagnation volume and the highest recorded value over one simulated cardiac or speed modulation cycle. Particle based metrics (exposure to shear stress $>50 \mathrm{~Pa}$ and PAS values) are derived using values computed for all injected particles ( $n=1,260,2,520$ and 3,780 for baseline, ventricular pulsatility, and speed modulation, respectively).

volume and $\sim 4 \%$ of HM3 fluid volume were characterized by stagnant flow (Table 1). Residual ventricular contractility and speed modulation sequences, and in particular transient acceleration of the pump flow rate, significantly reduced - but did not abate-flow stagnation (Figure 4, B, C, E, and F; Table 1). The high-speed phase of the HVAD LC reduced stagnation volumes by up to $57 \%$ compared with constant operating conditions. However, persistent flow stagnation was observed on the suction side of the blades and in the inner gap. In the HM3, stagnation volumes reduced up to $91 \%$ during the acceleration phase.

\section{Fluid Shear Stress}

Shear stresses in the HVAD with simulated constant speed reached up to approximately $150 \mathrm{~Pa}$ in the blade channels and, within the assumption of uniform blood viscosity, up to fivefold higher values in the narrow top gap (Figure 5A). Ventricular pulsatility and speed modulation did not significantly impact stress patterns and magnitude (Figure 5C), except for the top gap where shear rates, and therefore shear stresses primarily depended on the rotor speed and thereby decreased/increased during the low/ high speed phase of the LC. As a result, over $70 \%$ of the simulated platelets experienced high-shear stresses (>50 Pa) in the HVAD already under constant operating conditions; the exposure time profile was relatively unaffected by the LC dynamic speed modulation (Figure 6A). Shear stresses in the HM3 generated during constant operating conditions were significantly lower than in the HVAD and below $50 \mathrm{~Pa}$ in almost the entire pump (Figure 5B); almost 50\% of the flowing simulated platelets never experienced shear stresses $>50 \mathrm{~Pa}$ (Figure 6B). Stress levels dramatically increased during the acceleration and high-speed phases of the AP due to the onset of turbulence (Figure 5D): the contribution of the modeled turbulent Reynolds stress over total shear stress increased from $26 \%$ to $80 \%$; more than $90 \%$ of all injected particles experienced stresses $>50$ Pa (Figure 6B, Table 1), proportion that went up to $99 \%$ when considering particles injected just before the acceleration phase only.

\section{Shear-mediated Platelet Activation}

Analysis of PAS values provided insight into potential prothrombotic shear-mediated platelet activation imparted by

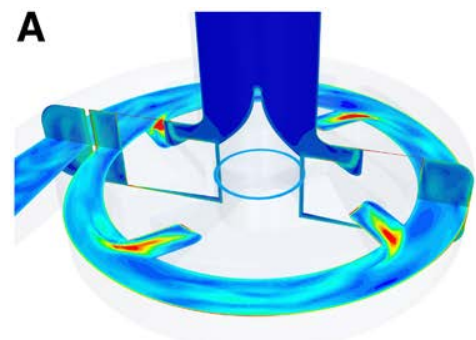

B
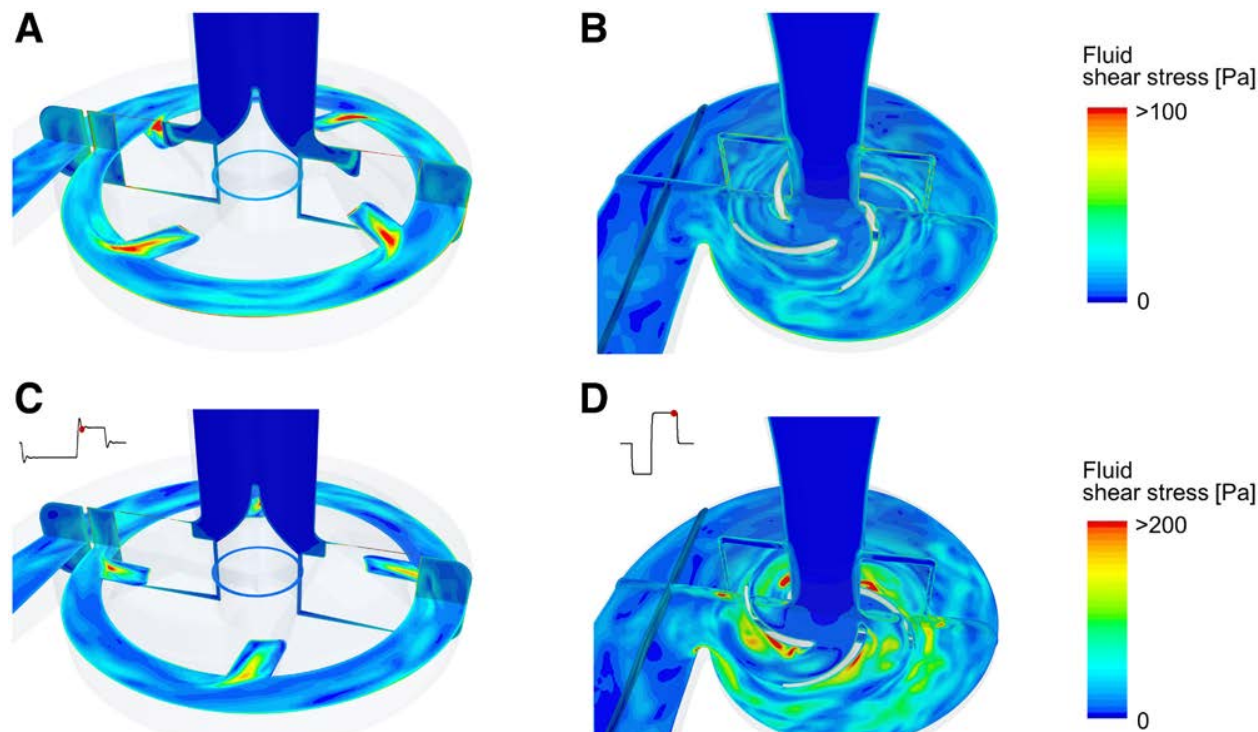

D

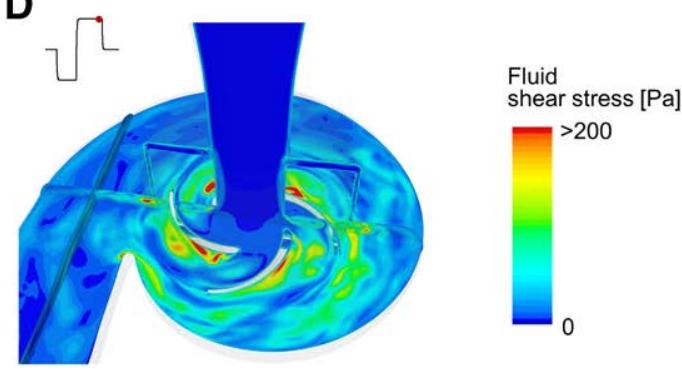

Figure 5. Distribution of fluid shear stresses in the HVAD and HM3 under constant operating conditions (A, B) and with enabled speed modulation sequences (C: Lavare Cycle, D: artificial pulse). Areas in red show regions with shear stresses above $100 \mathrm{~Pa}(\mathrm{~A}$ and $\mathrm{B})$ and above $200 \mathrm{~Pa}(\mathrm{C}$ and D). HM3, HeartMate3; HVAD, HeartWare ventricular assist device. 

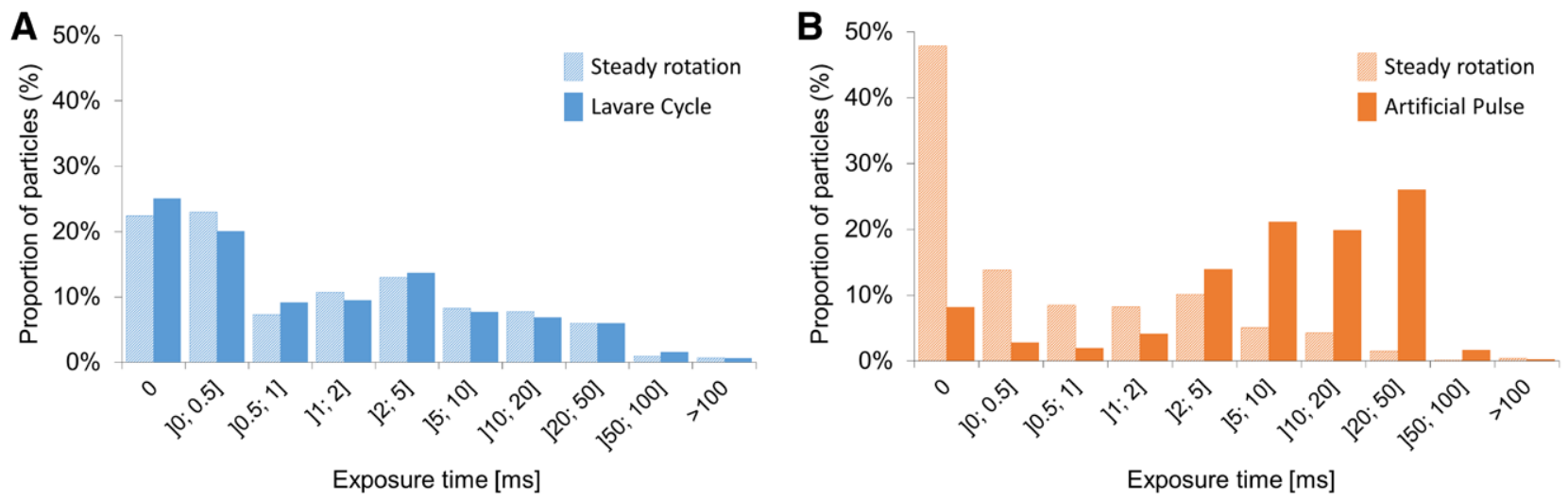

Figure 6. Comparison of the particle exposure time to fluid shear stress above $50 \mathrm{~Pa}$ in the HVAD (A) and HM3 (B) under constant operating conditions and with the LC or AP. The histograms were computed for a total of 1,260 particle tracks (one injection time point) in the HVAD and HM3 baseline scenario (steady rotation and fixed pressure head), and 3,780 particle tracks (three injection time points) for the LC and $A P$, respectively. To provide an integrated view on the effect of the different simulated conditions, all particle tracks were analyzed together. AP, artificial pulse; HM3, HeartMate3; HVAD, HeartWare ventricular assist device; LC, Lavare cycle.

speed modulation sequences (Figure 7). At constant operating conditions, numerical PAS was higher in the HVAD than in the HM3 (+66\% median value, Figure 7, Table 1). Passage through the top gap of the HVAD was especially detrimental as $62 \%$ of the particles with PAS values higher than 95th percentile of the PAS distribution traversed the top gap at least once. The HVAD LC did not yield any significant change in shearmediated platelet activation (Figure 7A, Table 1). Conversely, the HM3 AP significantly contributed to increase PAS values compared with constant operating conditions $(+124 \%$ median value; Figure $\mathbf{7 B}$, Table $\mathbf{1}$ ).

\section{DISCUSSION}

We coupled analysis of defined periodic flow pattern changes of rotor speed modulation sequences with juxtaposed instantaneous shear stress and platelet activation modeling to evaluate the impact of features of speed modulation sequences on thrombogenicity of two of the currently most used continuous-flow LVADs, that is, the HVAD and the HM3. Our analysis was aimed at overcoming limitations of previous studies that only analyzed pump operation with constant rotor speed. Moreover, we aimed at evaluating isolated effects of unsteady pressure head induced by residual cardiac pulsatility and of flow profile alterations driven by speed modulation sequences, to selectively characterize their prothrombotic impact.

Flow stasis is one of the pillars of Virchow's triad, increasing risk of platelet aggregation and deposition and clot formation. We quantified scalar washout and volumes of flow stagnation to compare the performance of the pumps under different operating conditions and identify pump regions potentially at higher thrombotic risk. Our results suggest that rotor speed modulation does not substantially enhance and expedite bulk pump washout, which was comparable irrespective of the pump being operated under constant or unsteady operating conditions. The 95\% washout time was also comparable across pumps, suggesting that, although the LC and the AP feature major differences in terms of magnitude and dynamics of rotor speed modulation $( \pm 200 \mathrm{rpm}$ over $3 \mathrm{sec} v \mathrm{v}$. $\pm 2,000 \mathrm{rpm}$ over $0.35 \mathrm{sec}$, respectively), the flow modulations
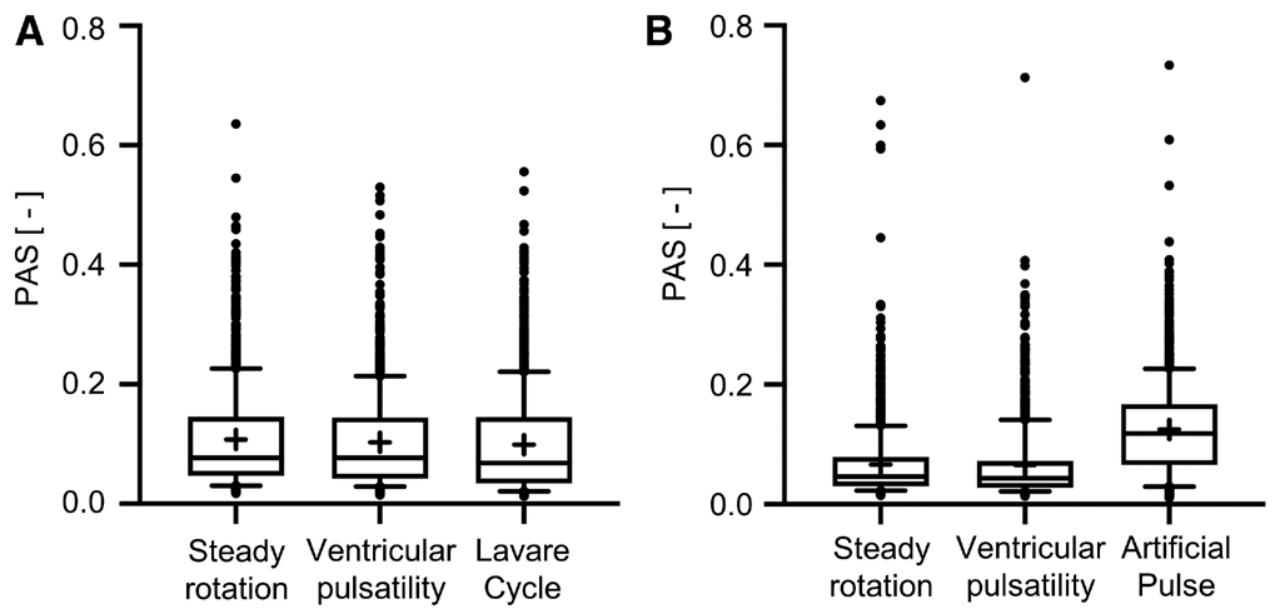

Figure 7. Box plot of numerical PAS values in the HVAD (A) and HM3 (B) plotted under constant and unsteady operating conditions. Particles were injected at the time points illustrated in Figure 2. To provide an integrated view on the effect of the different simulated conditions, all particle tracks were analyzed together. Bars indicate 25th, 50th, and 75th percentile; whiskers indicate 10th and 90th percentile; + indicates mean; • indicates outliers. AP, artificial pulse; HM3, HeartMate3; HVAD, HeartWare ventricular assist device; LC, Lavare cycle. 
they provide affect bulk pump washout only marginally. The latter rather depended on the mean pump flow rate, here maintained at $5 \mathrm{~L} / \mathrm{min}$ for all tested conditions. Transesophageal echocardiography velocities below $0.15-0.55 \mathrm{~m} / \mathrm{s}$ have been associated with increased risk of thrombus formation in patients with atrial fibrillation ${ }^{27-32}$; accordingly, flow stagnation was here defined as blood velocities below $0.2 \mathrm{~m} / \mathrm{s}$, a threshold that is comparable to that of previous studies that analyzed flow stagnation in LVADs. ${ }^{33}$ Furthermore, stagnation zones were evaluated $40 \mu \mathrm{m}$ away from the static housing or rotating impeller surfaces of the pump to exclude from the analysis inner regions of the boundary layer of the mesh of the pumps (details of the mesh are provided in the Supplementary Materials, Supplemental Digital Content 1, http://links.Iww. com/ASAIO/A564) and focus on regions of flow stagnation induced by the flow patterns in the core of the fluid regions. According to our results, volumes of flow stagnation were substantially reduced during the peak acceleration phases of the LC and AP ( $-57 \%$ and $-91 \%$, respectively). However, neither the LC nor the AP sufficed to completely eliminate stagnation zones, which seemed to be primarily dictated by specific design characteristics of the two pumps.

Although the exact threshold remains allusive, very low wall shear stresses (WSS $<1 \mathrm{~Pa}$, according to Hochareon et al. ${ }^{34}$ ) have also been suggested to contribute to the thrombotic risk. Under the investigated conditions, WSS below 1 Pa were almost absent: on both pump rotors, the sum of all areas with WSS $<1 \mathrm{~Pa}$ was accounted for less than $1 \mathrm{~mm}^{2}$ (data not shown), with a transient increase to $1-3 \mathrm{~mm}^{2}$ during the low flow phase of the HM3 AP, but individual low WSS regions were too spatially limited (few contiguous cells) and too transient to allow any inference about their actual thrombogenic impact.

In addition to characterization of thrombotic risk associated with flow stasis, we also show the effect of speed modulation on the fluid shear stresses generated within the devices. Our results revealed significant differences between the two pumps. Shear stresses in the primary flow path were significantly higher in the HVAD (Figure 5, A to C), where peak shear stress was located in the narrow clearance gaps and was therefore strongly associated with the impeller rotational speed. Accordingly, the LC only had marginal effect as it induces a relatively small change $( \pm 200 \mathrm{rpm})$ in rotational speed. Conversely, shear stresses in the HM3 increased substantially during the AP compared with those computed under constant operating conditions and appeared to be mainly related to turbulence introduced in the fluid flow during the impeller acceleration phase. These data suggest a prominent contribution of the high magnitude $( \pm 2,000 \mathrm{rpm})$ and sudden $(0.35 \mathrm{sec})$ speed change of the AP to the generation of prothrombotic conditions.

These results were further corroborated by analysis of metrics of platelet activation status. Indeed, while higher PAS values were computed in the HVAD than in the HM3 during steady pressure head and fixed speed simulations, the AP contributed to high increase of modeled shear-mediated platelet activation, bringing PAS values to levels even higher of those computed in the HVAD (Table 1). These data further suggest limited prothrombotic impact of the LC, and the potentially higher prothrombotic effect of the AP, which exposes platelets to high oscillating shear stress stimulation.
Our analysis also showed that although specific design features (geometrical and technological) of an LVAD differently influence structures of blood flow (e.g., blades geometry, internal gap dimensions, hydrodynamics, vs. fully magnetic levitation) and have a different impact on the device thrombogenic potential, replicating clinically relevant unsteady operating conditions is an essential requisite for robust and reliable evaluation-and eventually optimization — of pump performance.

Our results provide mechanistic insights into clinically reported outcomes, namely thrombotic and thromboembolic complications with the two pumps. Indeed, while the HM3 showed a reduced incidence of in-pump thrombosis, ${ }^{35}$ it did not perform consistently better than the HVAD in terms of neurologic complications secondary to thromboembolic events. ${ }^{36}$ These clinical data are consistent with our numerical characterization of pump thrombogenicity during clinically relevant operating conditions, which revealed that during the AP median PAS values in the HM3 where comparable to those of the HVAD (Table 1). These results suggest that the lower rate of HM3 in-pump thrombosis may not be attributed to actual lower pump thrombogenic potential but rather to the larger secondary flow channels and gap clearance between the housing and the blades, which facilitate propulsion of small thrombi downstream of the pump itself, before their growth, thus leading to thromboembolic events rather than pump thrombosis.

Our results are in accordance with and further extend results by Zimpfer et al., ${ }^{37}$ who reported fewer rates of stroke in HVAD patients who had an active LC, thanks to improvements in intraventricular flow field. Indeed, although we did not evaluate the effect of LC on the intraventricular hemodynamics, our simulations of the HVAD LC revealed (i) reduction of prothrombotic blood flow stagnation within the pump together with (ii) minimum/no increase of prothrombotic shear stress and overall metrics of platelet activation (Table 1). According to these results, we speculate that further improvement of clinical efficacy of the HVAD might be achieved via rationale optimization of the current speed modulation algorithm of the LC. In particular, further studies based on numerical simulation approaches as the one presented here are warranted to evaluate whether modifying magnitude and duration of the acceleration and deceleration phases of the LC, as well as increasing its activation frequency (i.e., more than once per minute) could provide further clinical benefits without worsening currently observed thrombogenicity. Similar considerations could be drawn for the HM3, which may benefit from computational model-guided optimization of the AP to reduce its thrombogenic impact. On the other hand, in an effort to improve hemocompatibility of LVAD speed modulation, we highlight the importance of properly balancing the reduction of possible detrimental effects associated with increased shear stresses and platelet activation with potential benefits of augmented pulsatility; indeed, consistent with the definition of HRAEs by Uriel et al., ${ }^{2}$ the evaluation of the effect of intermittent speed modulation on LVAD hemocompatibility should also incorporate analysis of its effect on pulsatility, and consequently on the development of aterovenous malformations, and, eventually, gastrointestinal bleeding.

Our analysis is inherently limited by the fact that calculating the entire dynamics of speed modulation while taking into account instantaneous changes in hemodynamic parameters is not feasible due to exorbitant computing power required to 
this end. That said, we carefully selected most relevant instants of the cycle (depending on the selected variable of interest) to provide robust assessment of how rotor speed modulation impacts hemodynamics and hemocompatibility. Furthermore, our analysis was only focused on characterizing the flow field within the pump and ignored further potential benefits of unsteady pump operation outside of the pump, such as increased intraventricular washout ${ }^{37}$ or changes in intraventricular pressure and its influence on aortic valve opening. Further studies are therefore warranted to evaluate the combined effect of intermittent speed modulation and native heartbeat but also of low and high pump speeds (thus low and high pump flows), on global hemodynamics and pump fluid dynamics. Yet, based on the results of the lumped parameters model of, ${ }^{16}$ we believe that the independent investigation of ventricular pulsatility and speed modulation already provides valuable insights into the in vivo situation. Further limitations are related to modeling of turbulent flow features with the unsteady RANS approach and the k- $\omega$ SST model (see Supplementary Materials, Supplemental Digital Content 1, http://links.Iww.com/ASAIO/ A564), which comes with a reasonable computational effort but does not fully resolve turbulence. ${ }^{38}$ Turbulence modeling in LVADs is a matter of ongoing research; still, we believe that our approach allowed us to identify major trends with regard to turbulent stresses relevant for the evaluation of thrombogenicity. Of note, the computed flow patterns in the HM3 during simulated unsteady operating conditions are coherent with previous experimental flow visualization studies. ${ }^{39}$ In addition, we did not include simulation of oscillations of the impeller position, which, from the one hand, is physically determined through interplay of magnetic and hydrodynamic forces and, on the other hand, is dependant from rapid changes in speed and consequent changes in pressure distributions and momentum forces acting on the impeller, and might therefore change during speed modulation. Nevertheless, we simulated a fixed impeller position to keep the computational effort in a manageable range. We also acknowledge that, although coherent with reported comparable prothrombotic platelet function measured via the PAS assay in HVAD and HM3 patients with active speed modulation regimes (i.e., the LC and the $\mathrm{AP}),{ }^{13}$ the numerical PAS index values requires further validation studies. Moreover, future studies are warranted to incorporate the evaluation of the effect of antithrombotic agents in limiting (yet without eliminating) the thrombotic risk. Finally, integrating the evaluation of high molecular weight vWF degradation, platelet damage (nonactivating or fragmentation), or platelet aggregation induced by speed modulation might extend the significance of our analysis.

\section{CONCLUSION}

We investigated clinically relevant operating conditions of current generation LVADs to evaluate the thrombotic risk of unsteady speed modulation regimens that are claimed to improve pump hemocompatibility. Our analysis couples evaluation of flow pattern alteration induced by speed modulation algorithms with tracking of generated fluid shear stress and modeling of platelet activation. Our results reveal partial improvements in pump hemodynamics but substantial increase in metrics of thrombogenicity caused by speed modulation. Our data are in good agreement with, and provide a possible mechanistic rationale for the clinically reported rates of thrombotic and thromboembolic complications with the HVAD and HM3. Our numerical platform represents a valuable tool for future design optimization of LVAD geometry and operating conditions, to further reduce HRAEs.

\section{REFERENCES}

1. Kormos RL, Cowger J, Pagani FD, et al: The society of thoracic surgeons intermacs database annual report: Evolving indications, outcomes, and scientific partnerships. / Heart Lung Transplant 38: 114-126, 2019.

2. Uriel N, Colombo PC, Cleveland JC, et al: Hemocompatibilityrelated outcomes in the MOMENTUM 3 trial at 6 months: A randomized controlled study of a fully magnetically levitated pump in advanced heart failure. Circulation 135: 2003-2012, 2017.

3. Mehra MR: The burden of haemocompatibility with left ventricular assist systems: A complex weave. Eur Heart J 40: 673-677, 2019.

4. Valerio L, Consolo F, Bluestein D, et al: Shear-mediated platelet activation in patients implanted with continuous flow LVADs: A preliminary study utilizing the platelet activity state (PAS) assay. Annu Int Conf IEEE Eng Med Biol Soc 2015: 1255-1258, 2015.

5. Consolo F, Sferrazza G, Motolone G, et al: Platelet activation is a preoperative risk factor for the development of thromboembolic complications in patients with continuous-flow left ventricular assist device. Eur J Heart Fail 20: 792-800, 2018.

6. Consolo F, Sferrazza G, Motolone G, et al: Shear-mediated platelet activation enhances thrombotic complications in patients with LVADs and is reversed after heart transplantation. ASAIO / 65: e33-e35, 2019.

7. Uriel N, Pak SW, Jorde UP, et al: Acquired von Willebrand syndrome after continuous-flow mechanical device support contributes to a high prevalence of bleeding during long-term support and at the time of transplantation. I Am Coll Cardiol 56: 1207-1213, 2010.

8. Bartoli CR, Restle DJ, Zhang DM, Acker MA, Atluri P: Pathologic von Willebrand factor degradation with a left ventricular assist device occurs via two distinct mechanisms: Mechanical demolition and enzymatic cleavage. J Thorac Cardiovasc Surg 149: 281-289, 2015.

9. Bartoli CR, Kang J, Restle DJ, et al: Inhibition of ADAMTS-13 by doxycycline reduces von Willebrand factor degradation during supraphysiological shear stress: Therapeutic implications for left ventricular assist device-associated bleeding. JACC Heart Fail 3: 860-869, 2015.

10. Valerio L, Tran PL, Sheriff J, et al: Aspirin has limited ability to modulate shear-mediated platelet activation associated with elevated shear stress of ventricular assist devices. Thromb Res 140: 110-117, 2016.

11. Valerio L, Sheriff J, Tran PL, et al: Routine clinical anti-platelet agents have limited efficacy in modulating hypershear-mediated platelet activation associated with mechanical circulatory support. Thromb Res 163: 162-171, 2018.

12. Consolo F, Pappalardo F: Letter by consolo and pappalardo regarding article, "comprehensive analysis of stroke in the long-term cohort of the MOMENTUM 3 study: A randomized controlled trial of the heartmate 3 versus the heartmate II cardiac pump." Circulation 140: e163-e164, 2019.

13. Consolo F, Pozzi L, Pieri M, et al: Influence of different antithrombotic regimens on platelet-mediated thrombin generation in patients with left ventricular assist devices. ASAIO / 66: 415422, 2020.

14. Chiu WC, Slepian MJ, Bluestein D: Thrombus formation patterns in the HeartMate II ventricular assist device: Clinical observations can be predicted by numerical simulations. ASAIO / 60: 237-240, 2014.

15. Bourque K, Cotter C, Dague C, et al: Design rationale and preclinical evaluation of the heartMate 3 left ventricular assist system for hemocompatibility. ASAIO / 62: 375-383, 2016.

16. Wiegmann L, Thamsen B, de Zélicourt D, et al: Fluid dynamics in the HeartMate 3: Influence of the artificial pulse feature and residual cardiac pulsation. Artif Organs 43: 363-376, 2019. 
17. Chivukula VK, Beckman JA, Prisco AR, et al: Left ventricular assist device inflow cannula angle and thrombosis risk. Circ Heart Fail 11: e004325, 2018.

18. Selmi M, Chiu WC, Chivukula VK, et al: Blood damage in left ventricular assist devices: Pump thrombosis or system thrombosis? Int J Artif Organs 42: 113-124, 2019.

19. Zhang Y, Gao B, Yu C: The hemodynamic effects of the LVAD outflow cannula location on the thrombi distribution in the aorta: A primary numerical study. Comput Methods Programs Biomed 133: $217-227,2016$

20. Aliseda A, Chivukula VK, Mcgah P, et al: LVAD outflow graft angle and thrombosis risk. ASAIO / 63: 14-23, 2017.

21. Mahr C, Chivukula VK, McGah $\mathrm{P}$, et al: Intermittent aortic valve opening and risk of thrombosis in ventricular assist device patients. ASAIO / 63: 425-432, 2017.

22. Kumar J, Elhassan A, Dimitrova G, Essandoh M: The lavare cycle: A novel pulsatile feature of the HVAD continuous-flow left ventricular assist device. / Cardiothorac Vasc Anesth 33: 11701171,2019

23. Wever-Pinzon O, Selzman $\mathrm{CH}$, Drakos SG, et al: Pulsatility and the risk of nonsurgical bleeding in patients supported with the continuous-flow left ventricular assist device HeartMate II. Circ Heart Fail 6: 517-526, 2013.

24. Purohit SN, Cornwell WK III, Pal JD, Lindenfeld I, Ambardekar AV: Living without a pulse: The vascular implications of continuousflow left ventricular assist devices. Circ Heart Fail 11: e004670, 2018.

25. Consolo F, Sheriff I, Gorla S, et al: High frequency components of hemodynamic shear stress profiles are a major determinant of shear-mediated platelet activation in therapeutic blood recirculating devices. Sci Rep 7: 4994, 2017.

26. Thamsen B, Plamondon $M$, Granegger $M$, et al: Investigation of the axial gap clearance in a hydrodynamic-passive magnetically levitated rotary blood pump using X-ray radiography. Artif Organs 42: 510-515, 2018.

27. Shively BK, Gelgand EA, Crawford MH: Regional left atrial stasis during atrial fibrillation and flutter: Determinants and relation to stroke. J Am Coll Cardiol 27: 1722-1729, 1996.

28. Goldman ME, Pearce LA, Hart RG, et al: Pathophysiologic correlates of thromboembolism in nonvalvular atrial fibrillation: I. Reduced flow velocity in the left atrial appendage (the stroke prevention in atrial fibrillation [SPAF-III] study). J Am Soc Echocardiogr 12: 1080-1087, 1999.

29. Zabalgoitia M, Halperin JL, Pearce LA, Blackshear JL, Asinger RW, Hart RG: Transesophageal echocardiographic correlates of clinical risk of thromboembolism in nonvalvular atrial fibrillation.
Stroke prevention in atrial fibrillation III investigators. J Am Coll Cardiol 31: 1622-1626, 1998.

30. Panagiotopoulos K, Toumanidis S, Vemmos K, Saridakis N Stamatelopoulos S: Secondary prognosis after cardioembolic stroke of atrial origin: The role of left atrial and left atrial appendage dysfunction. Clin Cardiol 26: 269-274, 2003.

31. Fatkin D, Kuchar DL, Thorburn CW, Feneley MP: Transesophageal echocardiography before and during direct current cardioversion of atrial fibrillation: Evidence for "atrial stunning" as a mechanism of thromboembolic complications. I Am Coll Cardiol 23: 307-316, 1994.

32. Handke M, Harloff A, Hetzel A, Olschewski M, Bode C, Geibel A: Left atrial appendage flow velocity as a quantitative surrogate parameter for thromboembolic risk: Determinants and relationship to spontaneous echocontrast and thrombus formation-A transesophageal echocardiographic study in 500 patients with cerebral ischemia. I Am Soc Echocardiogr 18: 1366-1372, 2005.

33. Granegger $M$, Thamsen B, Schlöglhofer $T$, et al: Blood trauma potential of the HeartWare ventricular assist device in pediatric patients. J Thorac Cardiovasc Surg 159: 1519-1527.e1 2020

34. Hochareon P, Manning KB, Fontaine AA, Tarbell JM, Deutsch S: Correlation of in vivo clot deposition with the flow characteristics in the $50 \mathrm{cc}$ penn state artificial heart: A preliminary study. ASAIO / 50: 537-542, 2004

35. Mehra MR, Uriel N, Naka Y, et al; MOMENTUM 3 Investigators: A fully magnetically levitated left ventricular assist device-final report. N Engl J Med 380: 1618-1627, 2019.

36. Li S, Beckman JA, Cheng R, et al: Comparison of neurologic event rates among HeartMate II, HeartMate 3, and HVAD. ASAIO 66: 620-624, 2019

37. Zimpfer D, Strueber M, Aigner P, et al: Evaluation of the HeartWare ventricular assist device Lavare cycle in a particle image velocimetry model and in clinical practice. Eur J Cardiothorac Surg 50: 839-848, 2016

38. Torner B, Hallier S, Witte M, Wurm FH. Large-eddy and unsteady reynolds-averaged navier-stokes simulations of an axial flow pump for cardiac support. in Proceedings of the ASME Turbo Expo 2017: Turbomachinery Technical Conference and Exposition, Vol. 2B. Turbomachinery, Charlotte, NC, June 2630, 2017.

39. Thamsen B, Gülan U, Wiegmann L, et al: Assessment of the flow field in the HeartMate 3 using three-dimensional particle tracking velocimetry and comparison to computational fluid dynamics. ASAIO / 66: 173-182, 2020. 